\title{
Nasogastric tube location: a diagnostic dilemma
}

\author{
Pradipta Bhakta, MD, MNAMS, FCAI, EDRA 무 Timothy Keady, MCAI • \\ Brian O'Brien, FCARCSI, FJFICMI, FCICM, ANZ
}

Received: 4 February 2018/Revised: 6 February 2018/Accepted: 6 February 2018/Published online: 21 February 2018

(C) Canadian Anesthesiologists' Society 2018

A 79-yr-old woman was admitted to hospital with presumed respiratory sepsis. Her past medical history included hypertension, coronary artery disease, and a hiatus hernia. She was initially managed with noninvasive ventilation, antibiotics, and vasopressors. To allow enteral feeding, a wide-bore (14 Fr) nasogastric tube (NGT) was inserted, and its position was provisionally confirmed using auscultation of injected air, although nothing could be aspirated from it. Chest radiography (CXR) was performed for definitive confirmation of the NGT position before initiating feeding, which revealed that the NGT tip appeared to be in the right lung base (Figure). Assuming misplacement, the NGT was removed, and the procedure was abandoned. Later the same day, the patient's respiratory status deteriorated, and she was intubated and ventilated.

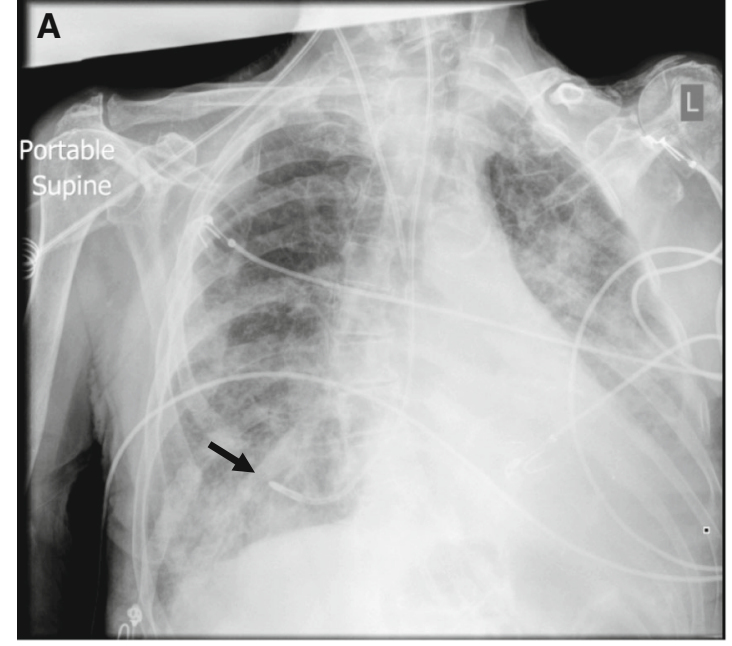

Figure A) Chest radiograph showing a nasogastric tube overlying the right lung field. The black arrow indicates the tip of the nasogastric tube that appears to be in the right hemi-thorax. B) Computed tomography

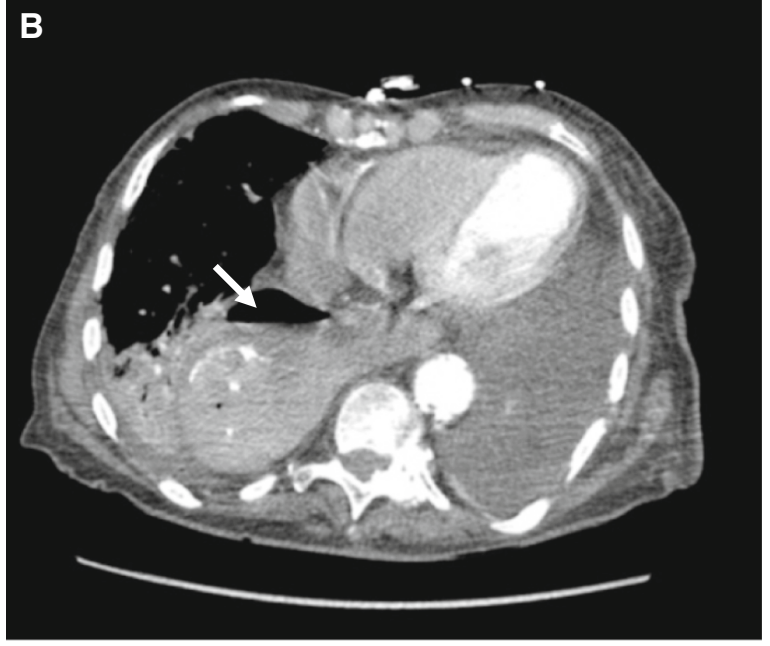

image of the chest showing a large hiatal hernia (arrow) extending into the right hemi-thorax. The images were used with the patient's permission
P. Bhakta, MD, MNAMS, FCAI, EDRA ( $\square)$.

T. Keady, MCAI - B. O'Brien, FCARCSI, FJFICMI, FCICM, ANZ

Department of Anaesthesia and Intensive Care, Cork University

Hospital, Cork, Ireland

e-mail: bhaktadr@hotmail.com 
Computed tomography (CT) subsequently showed a large hiatus hernia extending into the right hemi-thorax (Figure). Hence, it was deemed likely that the initial NGT had not been placed in the right lung, but instead was in the hernia itself. A new NGT was successfully reinserted later that evening following admission to the intensive care unit.

Although CXR is still considered the gold standard for confirming NGT placement, the presence of a hiatus hernia, a common finding in elderly patients, may lead to errors in interpretation. In these cases, consideration should be given to alternative investigations such as $\mathrm{pH}$ testing of aspirated fluid or ultrasonography of the stomach to confirm proper NGT positioning.

Conflicts of interest None declared.

Editorial responsibility This submission was handled by Dr. Hilary P. Grocott, Editor-in-Chief, Canadian Journal of Anesthesia. 